\title{
SURVEY ON EFFICIENT POWER UTILIZATION IN WIRELESS SENSOR NETWORKS
}

\author{
Premkumar $\mathbf{A}^{1}$ \\ ${ }^{1}$ Student, Acharya Institute of Technology, Bangalore
}

\begin{abstract}
This paper has addressed the distributed energy/power change in Wireless Sensor Network (WSN). With new technologies WSN evolving with efficient and new features, because of this live field for research and development oriented work have turn out to be more in the field of WSN. The main components of WSN are, each node is deployed with required software and hardware system and limited energy usage in terms of power consumption for particular work assigned to the sensor hub which uses energy for the work. Each hub is embedded with resources like memory, microcontroller, and antenna. Where memory is use for storing and forwarding of data, whereas microcontroller is used for processing and providing data security using encryption and also used to maintain and updating of routing protocols. Antenna is used for signal detection and for transmission (receiving and broadcasting signals). Sensor hubs uses large amount of energy, to have life time of node in the network .it is important to increase its lifetime in the group. There has been enormous effect to increase the life of hub further in the network by providing new techniques and changes to various sensor node layer for better life in the network group. In paper different approaches have been come upon based on usage of energy by the sensor.
\end{abstract}

Keywords: Ad-Hoc, MANET, VANET, FANET, Power utilization, Routing protocols, lifetime, low power WSN, IEEE Standards, Mobile sink.

\section{INTRODUCTION}

In Wireless Sensor Network (WSN), distributed network points in wireless mesh network helps in exchanging encrypted data. Using AES and DES algorithms can perform encryption and decryption on information which convert readable information into unreadable format for the security of data which is exchanged over the network, the deployment of sensor hubs was more focused towards military and civilian applications [1].

Sensor networks are used in monitoring and controlling in the fields like industrial process inspection, health examining, control over traffic, home mechanization, healthcare application.

WSN are classified into: -

Ad Hoc networks [1],Ad-Hoc Network are decentralized kind of remote system. The system is specially fixed on the grounds that it doesn't depend on a prior framework, for example, adjustments in wired systems (infrastructure) remote systems. Rather, every hub takes part in routing by sending information for different hubs, so the assurance of which hubs forward information is made strongly on the premise of system availability and routing algorithms being used. Inthis set-up all network points are static and communication takes place between nodes and base station for exchanging controlled signals.

The significant information primarily transmitted through network points-sink-network points.
MANET [1], MANET systems are self-organizing, dynamic systems in which hubs are allowed to move. Remote systems do not have the complexities of framework setup and organization, empowering devices to make and join in network setup network nodes has mobility this type of arrangement is based on routing technologies implemented in each node in the network group.

Further in the system communication takes place between network hubs and base station for having controlling over the setup.

VANET [1], uses the principles of MANET, In Vehicular Ad Hoc networks all the nodes are being embedded in the vehicles for exchanging the controlled data.

VANET are used for communication among vehicles and roadside equipment. smart vehicular ad hoc networks (InVANETs) are a variety of artificial intelligence that helps vehicles to behave in intelligent manners during vehicle-tovehicle collisions, accidents. Vehicles are usingradio waves to communicate with each other, creating communication networks instantly on-the-fly while vehicles are moving on the roads.

In this type of network data exchange takes place between nodes. Base station is not required because each node will behave as normal nodes as well as sink nodes in the network.

FANET [1],Flying Ad Hoc Networks. In this type of network, all network points are distributed in the sky for monitoring and for exchanging the controlled data/information each hub is deployed with routing updates and routing algorithms. 
All distributed hubs will be controlled by the cluster head and base station, base station plays important role in the field of FANET to control over the nodes, scenarios like to check the energy levels of nodes, for clustering and cluster head selection. With this advantages FANET are used in military applications Figure 1 .

Wireless Sensor Network, are classified as shown in the below table 1 .

Table 1: Applications of wireless sensor network

\begin{tabular}{|l|l|}
\hline Wireless networks & Applications \\
\hline Ad-Hoc & Cell phones, Home automation \\
\hline MANET & Cell phones \\
\hline VANET & vehicles and roadside equipment \\
\hline FANET & Military \\
\hline
\end{tabular}

The interior sensor node components are shown in the figure2, contain transceiver, microcontroller, external memory, power resource and sensor information scheming and functionality of every components in the node is controlled by microcontroller.

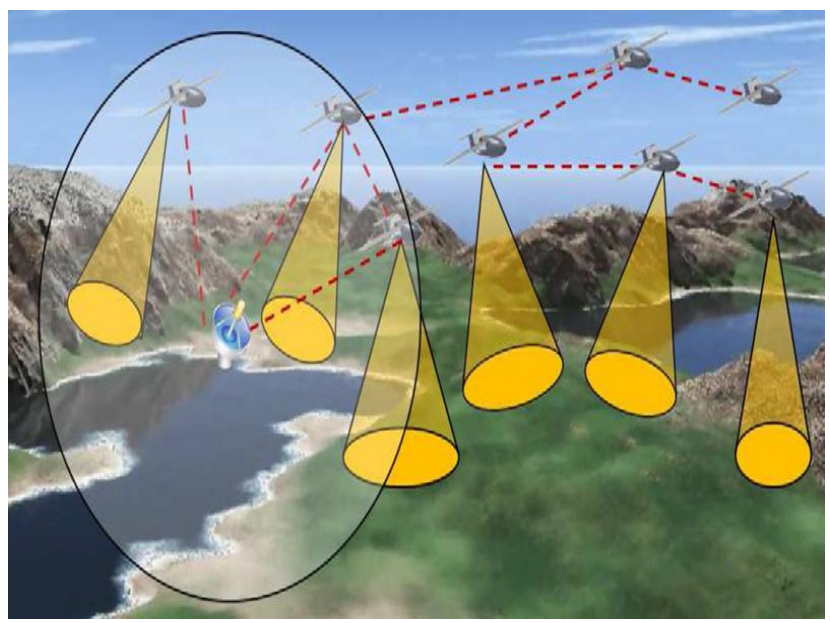

Fig 1: FANETS in military application [1]

Microcontrollers are suitable for sensor node and embedded system

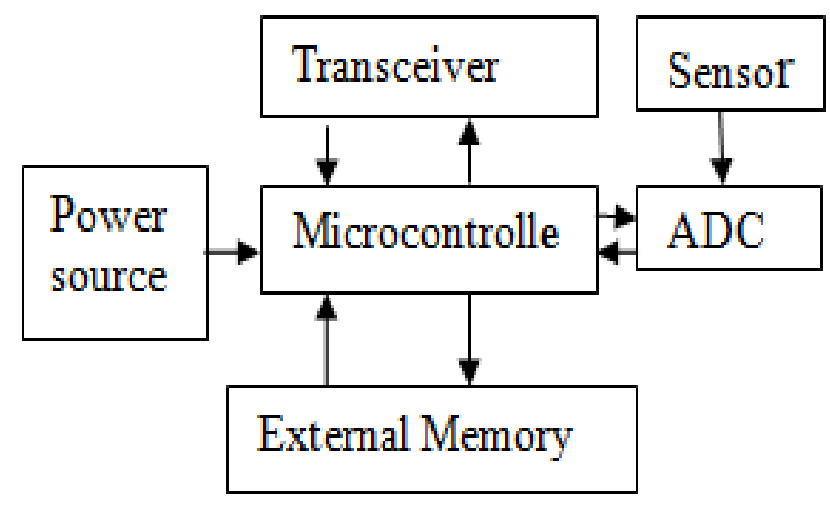

Fig 2: core components of sensor nodes
In this paper, section 2discussvarious WSN standards and protocols in wireless sensor networks for low power utilization. section 3 constituents for discrete use of energy.in section 4Multi network models, in section 5. Challenges have been discussed in. section 6. Various related work carried by the experts.

\section{STANDARDS AND PROTOCOLS IN WIRELESS SENSOR NETWORKING FOR LOW POWER UTILIZATION}

The IEEE 802.11 group of models is usedfor theWireless LAN (WLAN) or Wireless Interconnects Network (WMN). It is usually containing of channels, work with the help switches and regulars [5]. The IEEE 802 standard that are dedicated for this paper is the IEEE 802.15.4, the Low-Rate Wireless Private Area Network (LR-WPAN) standard, which is generally used for the frameworks with constrained calculation and power resources, for example, on account of the isolated sensor organize (WSN) applications. The IEEE 802.11 standard have higher implementation difference with IEEE 802.15.4 standard as far as broadcast run, information rate and throughput. The IEEE 802.11 has higher power utilization than the IEEE 802.15.4[5]. Table2 shows essential assessment of 802.15.3 (High-Rate Wireless Private Area Network, HR-WPAN) versus the 802.15.4 (LR-WPAN) [5].

For the radio communication, the IEEE 802.15.4 standard uses three repetition groups, $868 \mathrm{MHz}, 915 \mathrm{MHz}$ and 2.4 GHz. distinctive information rates are accessible because of the different physical independence in each band; with 20 kbps, $40 \mathrm{kbps}$ and $250 \mathrm{kbps}$ for $868 \mathrm{MHz}, 915 \mathrm{MHz}$ and 2.4 $\mathrm{GHz}$ individually are used I IEEE 802.15.4 [5].

The essential transmission control for the IEEE 802.15.4 standard for each device is ranges just between 2 - 3 diameter. In late frameworks, the framework on-a-chip Silicon (Soc) usage exhibit extremely coordinated along with minimum effort arrangement, which is fit for taking care of the required components [5].

A few isolated advancements have been formed that are reasonable for remote sensor systems, ZigBee/RF4CE, ANT, ANT+, Nike+, and Bluetooth (Classic and BLT/BT v4.0). Every convention has its own preferences and inconveniences for WSN and is extremely focused with each other [5].

Table 2: Protocols and Standards

\begin{tabular}{|l|l|l|}
\hline Parameters & $\begin{array}{l}802.15 .3 \\
\text { HR-WPAN }\end{array}$ & $\begin{array}{l}802.15 .4 \\
\text { LR-WPAN }\end{array}$ \\
\hline Distance & $10-100 \mathrm{~m}$ & $\sim 10 \mathrm{~m}$ \\
\hline Data speed & $1 \mathrm{Mbps}$ & $<0.25 \mathrm{Mbps}$ \\
\hline Power utilization & Low & Very low \\
\hline Size & Small & Smallest \\
\hline Complications & High & Low \\
\hline Expenditure & Low & Very low \\
\hline
\end{tabular}


ZigBee is a insignificant attempt, low power remote work organizing standard. "ZigBee" name is derived from which 'miscall crisscross examples honey bees make while moving between blossoms when gathering dust [5]. This is like the undetectable networks of associations framing in a remote system, through which bundles are directed [5]. The ZigBee standard determines a most extreme of $250 \mathrm{kbps}$ information rate.

Bluetooth Low Energy (BLTE) is a modern convention thoroughly composed through a lot of regard for accomplish low power utilization as well as superior [8].

Bluetooth v4.0 forum was discharged in year 2010, predominantly for BLTE. Bluetooth 4.2 release in year 2014, presents various key mechanism for supporting the Internet of Things (IoT). Bluetooth 4.2 exchanges information much quicker by expanding the limit of the Bluetooth [4][5].

\section{CONSTITUENT FOR DISCRETE USE OF}

\section{ENERGY}

Majormodern techniques for taking care of energy utilization problem in the hubs are explored. Their strategies are classified by means of Information diminishment, Broadcasting enrichment, rest/wake nodes, utilizing portable sink routing with energy effectiveness allowing for extra energy and respond for charging. This grouping is utilized for capable utilization of strength [4].

\subsection{Broadcasting Enrichment}

The word broadcasting refers to the technology for efficient transferring methods, for transferring data to all the users concurrently. Where broadcasting is full duplex transferring and receiving is done simultaneously of encrypted data in the network. The main important feature is analog signals are converted into digital and transfer through the network channels, broadcasting is classified into unicasting, broadcasting, multicasting, recasting.

Radio unit are primary segments for releasing battery strength in sensor hubs experts utilized special techniques, for example, programming, regulation schema, determining level of transform control and located reception apparatus for withdrawing energy disaster taking into consideration distant communication [4].

\subsection{Information Diminishment}

Information refers to the gathering of processed data is used to broadcast over the set-up. Information to be protected over the network the concept of encryption is used for providing information security in the network for data transmission. Information may contain any of the following formats, i.e. text files, image files, video files, audio files. To perform encryption to the following formats in WSN each node may use large amount of power resources to perform data encryption over the network [4].
One of alternate method for losing position energy utilization is decreasing measure of information in time of distribution to sink; two possible ways are introduced 1sending worthless sample 2-restriction in estimation transfer that could be adjusted normally. Since in the two situations, transmitting information and utilizing energy supply is finished. Strategies utilizing for this undertaking are [4]:

- Accumulation/aggregation: information from each node is been accumulated and transmitted to the required nodes.

- Adaptable sampling: each node has the ability to change its positions, and can adapt itself according the physical conditions of the environmental

- Network coding: each network points in the network has been deployed with required software. Routing and network coding to have secure communication between each node.

- Information compression: Information accumulated from each nodes are beaning compressed at particular point. The accumulated data/information to have light weight communication between nodes.

\subsection{Rest/Wake Modes}

Rest/Wake nodes have been introduced in the field of WSN. Rest/Wake Technique particular set of group nodes are made active an ideal in the network, whenever active nodes are in busy with task performance the ideal nodes are in the sleep mode, until the active nodes complete the task if the active node goes down it means when power drains in the active node, then ideal node wake up from sleep mode and participate in the task completion

Ideal modes are primary hotspot for energy utilization in radio part. The point of rest/wake modes is to coordinate circumstance of hub action to additional strength, utilizing rest mode in radio. A few techniques have been proposed [4].

- Duty cycling plans: each node in the network has the responsibility to perform particular task at given time, it is decided by the duty cycle software present in the network points.

- Passive wake up radio: nodes which are in the sleep mode is made to work at the specific time, when active nodes losses the energy levels then passive wake up radio signals activates the sleeping nodes in the network

- Topology control: all the nodes are controlled by the sink node and the cluster nodes. All the nodes are distributed in the network using the topology set by the sink nodes. all the nodes are controlled by the cluster head, all cluster head is under the control of sink nodes.

\subsection{Utilizing Portable Sink}

Soaks in sensor organize, are notable for incorporating simple data from sensor hubs. Preparing this simple data and offer them to another client. At the end of the day, amongst client and sensor hubs that could be static or dynamic in sensor systems. Late have demonstrated that portable sink is more helpful than static sink is regularly situated in a foreordained condition. Multitalented sinks prompt 
increasing sensor position lifetime and diminishing strength utilization in sensor hubs and due to incessantly movement organize keeping it from making hotspot flexible sinks developments and methodologies of being portable utilizing these models are classified in three classes [4].

- Irregular development: irregular development is possible if the nodes are portable and light weight.

- Unsurprising development: because of light weight and portable it helps to have unsurprising design and development of nodes.

\subsection{Routing With Energy Effectiveness}

In WSN every node participates in the network for completing the task. For Monitoring and exchanging of information, to exchange the information between the nodes and sink node(base station) we need routing information/routing protocols, exchanging tasks place between node-to-node and node-to-base station. Based on that routing protocols are been classified, Proactive, Reactive and hybrid routing protocols in this context we used Power aware routing protocol for each hub that participate in the network. Because of PAR each node consumes less amount of power and improves the performance of routing between nodes and the sink nodes [4].

Routing is extra load that can genuinely release energy source. In particular, in multi way plots, more focus is around close hub to sink. Since these hubs must track more packages contrasting with different hubs, battery release happens more quickly. In the additional, naming the systems for energy sparing in this routing. For more extensive examinations, these systems of energy careful routing conventions might be studied [4].

\section{NETWORK MODELS}

In this scenario all the distributed nodes benign used for the purpose of monitoring along with have controlling over the region, number of node are deployed [1][2]. Based on the distance and coverage area of the field to be controlled. Larger the area deployment of sensor hubs also increased in numbers, further deployment of hubs are carried after prototype model is made, prototype refers to the sample/dummy model is used before the actual development of the node, in prototype model it helps to check whether the required parameters are provided to the nodes are not, in dummy model results may not be accurate but can consider at that particular time, to create sample model using some simulator i.e.NS-2, NS-3[1][2][3]. Network simulator 2 and 3 , which uses TCL and C++ languages TCL, is tool command language, and $\mathrm{C}++$ is used as back end. TCL where script is written at particular time interval the event should occur, where as $\mathrm{C}$ is used to write code for energy levels, routing, data transfer, throughput, delay and number of nodes to be deployed [3].

For network demonstrating, it is conventional that an arrangement of sensor hubs is arbitrarily arranged to environmental checking. Figure 3 demonstrates a system show in which sink hub is moving along extent or edge of circle [3].

Then again, sink development in defeats which are featured in MDCA, has less effectiveness contrasted with defeats which sinks go while traveling through the system.

In this paper, clarified about last development of sink; sinks are scheduled forward concentric circles around the system condition.

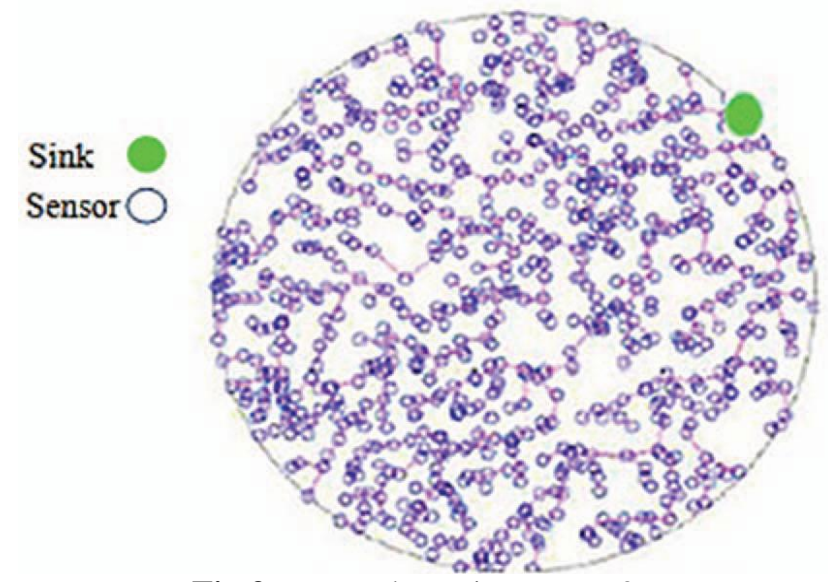

Fig 3: Network Environment [3].

Curved development could happen with different length and sinks are with various separations from each other, we consider position of sinks in sweep of 25,50 , and $100 \%$ as the base position of sink which is appeared in the figure $4[3]$.

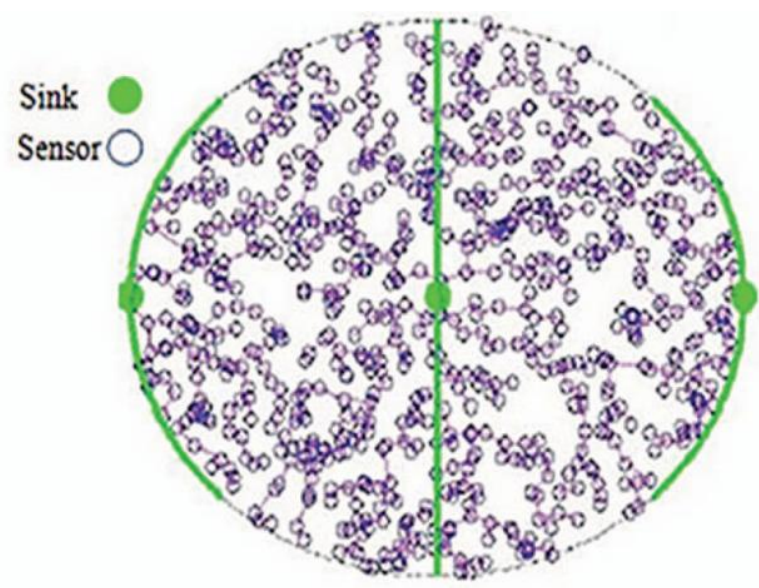

Fig 4: [3] Sink Movement in the MDCA method

\section{CHALLENGES}

Wireless sensor networks have more challenges, one of the most important challenge is power utilization in the sensor node for data exchange and have control

Major challenges in WSN design to overcome less resource constraints and guarantees data transmission and network life time, monitoring and end to end delay must be maintained. 
Over the network there is a need of power resources. Other challenges like routing which is also an issue because for finding the particular route for data exchange it requires energy utilization, and also some challenges in WSN like security, for defending the external attack in the network which may be like malicious node, black hole attack, gray hole attack etc. which made the analysts to work in the field of WSN, this paper is more focused on the energy utilization and more detailed work is carried.

\section{RELATED WORK}

Numerous analysts have tended to remarkable strategy for decreasing energy utilization with increasing system life span among the remote sensor systems. In work out a heuristic calculation toward locate the greatest amount of network points spreads a subset of hubs which container totally cover up the whole investigation region. Heuristic try to cover handle that are secured by few sensors and tries to maintain a strategic distance from very high utilization of individual sensors which wrap meagerly secured field [2].

L.F. Wolsey conveyed the scientific encoding show which depended on top of a deterioration and large issue [2]. It comprises of navigation and booking issues, by a segment period plan. The excited advance is formulated towards violence sensor spatial overload; it enhanced scales headed for enormous organizes also is turned out in the direction of compelling as far as execution in a few situations.

Keith Hellman and Michael Colgrosso's think about centers on the significant strength efficiency issues in remote sensor systems [2]. Establishment less systems that requires various jump for interfacing every one of the hubs among every one other. Mix of erect layer and criticality of strength utilization are primary attributes of remote sensor organizes to impel their plan.

Detachment of system capacity in layers is described as the first indulgence in systems administration [2]. A study on scope issues in remote sensor systems is given in. They ordered scope issues region scope to cover a region and point scope, to cover an arrangement of targets, and scope issues to decide the maximal help/break way that navigates a sensor field.

\section{CONCLUSION}

Fact that utilizing different adaptable sink for remote sensor systems has its own impediment; it could be a coherent determination. Utilizing, position life is expanded. In this examination, researched MDCA calculation in which portable sink moves along foreordained defeat to gather information from sensor hubs. In paper endeavored to choose district of sinks development all the more specifically. so as different to scheduled forward the edges or width of system, considered sink to move in driven around with range, utilized duty cycle of rest/wake mode to build organize lifetime and power utilization control. In each cycle $10 \%$ of sensor hubs with the less energy in entire system, get snoozing and this is proceeded until end of system runtime

\section{REFERENCES}

[1] Bekmezci, Ilker, Ozgur Koray Sahingoz, and Şamil Temel. "Flying ad-hoc networks (FANETs): A survey." Ad Hoc Networks 11.3 (2013): 1254-1270.

[2] Boubekri, Amine, Wessam Ajib, and Mounir Boukadoum. "EAM: Energy Aware Mobility over wireless sensor networks." Electrical and Computer Engineering (CCECE), 2017 IEEE 30th Canadian Conference on. IEEE, 2017.

[3] Singh, Jasvinder, and Er Vivek Thapar. "Intrusion Detection System in Wireless Sensor Network." International Journal of Computer Science and Communication Engineering 1.2 (2012): 76-80.

[4] Nazar, Navid Haghighat, and Mohammad Reza Heydarinezhad. "Enhancement lifetime of wireless sensor networks with mobile sink managed and improved routing and control Power Consumption." Knowledge-Based Engineering and Innovation (KBEI), 2015 2nd International Conference on. IEEE, 2015.

[5] Sharma, Amit, and Girma S. Tewolde. "Considerations in low power wireless sensor networks." Electro/Information Technology (EIT), 2015 IEEE International Conference on. IEEE, 2015. 